\title{
SphericalMesh: A Novel and Flexible Network Topology for 60GHz-based Wireless Data Centers
}

\author{
Yang Li, Fan Wu, Xiaofeng Gao, and Guihai Chen \\ Shanghai Key Laboratory of Scalable Computing and Systems \\ Department of Computer Science and Engineering \\ Shanghai Jiao Tong University, China \\ Email: Miracle302@sjtu.edu.cn, \{fwu,gao-xf, gchen\}@cs.sjtu.edu.cn
}

\begin{abstract}
Data center networks are becoming increasingly important for computing industry. By exploiting millimeter wavelength wireless technology (e.g., 60G Hz), data centers are going to be more convenient for the interconnection of different components without complex wiring. $60 \mathrm{GHz}$ technique has been widely studied, and great progress has been achieved indeed. However, there are two significant challenges unsolved in $60 \mathrm{GHz}$-based wireless data centers networks: link blockage and long network diameter. In this paper, we propose a new network topology, called Spherical Mesh, to improve the performance of wireless data center networks. Spherical Mesh can greatly mitigate link blockage by putting antennas at different heights, and decrease the network diameter by splitting the whole mesh into several equivalent units. Furthermore, we have implemented Spherical Mesh and evaluated its performance in different scenarios. Our evaluation results show that Spherical Mesh achieves minimal routing distance and guarantees the balance of workload.
\end{abstract}

\section{INTRODUCTION}

The increasingly fast development of data center networks require scalability, low cost effectiveness, high throughput and low oversubscription. As a result, several works (e.g., [2], [3]) have investigated the techniques for deploying more efficient data centers and network topologies. Unfortunately, most of them are based on wired topology, causing complex wiring and difficult configuration. To overcome these problems, wireless

This work was supported in part by the State Key Development Program for Basic Research of China (973 project 2014CB340303 and 2012CB316201), in part by China NSF grant 61422208, 61472252, 61272443 and 61133006, in part by Shanghai Science and Technology fund 12PJ1404900 and 12ZR1414900, and in part by Program for Changjiang Scholars and Innovative Research Team in University (IRT1158, PCSIRT) China. The opinions, findings, conclusions, and recommendations expressed in this paper are those of the authors and do not necessarily reflect the views of the funding agencies or the government.

$\mathrm{F} . \mathrm{Wu}$ is the corresponding author. communication technology has become a natural choice because of its flexibility in setup and low cost in deployment.

In recent years, $60 \mathrm{GHz}$ wireless communication technology has been exploited to replace or supplement the underlying wired topology. This recently popularized wireless technology has enabled high speed data transmissions that are suitable for data center networks. Several topologies (e.g., [4], [5], [9], [17]) have been proposed to achieve some attractive properties, such as more available links between nodes, high bandwidth and less completion time for network traffic.

However, these existing topologies have some limitations. The first one is link blockage, which happens when communication between nodes is blocked by another node. Although the novel topology proposed by Zhou et al. [16], 3D beamforming, solves this problem by bouncing off signal from the ceilings, it can only work well under the premise of flat and relatively low ceilings. The second drawback is the long network diameter. The network diameter, defined as the longest of all the shortest paths in a network, is an important performance metric of routing algorithm. Despite several years of studies on the network diameter in wired networks [2], [7], few work considers this issue in the area of wireless data center networks. To overcome these drawbacks, we investigate the characteristic of $60 \mathrm{GHz}$ signal's transmission, and propose Spherical Mesh, a novel and flexible wireless topology for data centers. In Spherical Mesh, we put antennas on several spheres to prevent signal from being blocked and reduce the network diameter. Our contributions are listed as follows.

- First, we propose a practical and flexible wireless topology, called Spherical Mesh. This topology can mitigate link blockage without making constraints 
on the ceilings.

- To the best of our knowledge, we are the first to consider the network diameter for wireless data center networks. We also propose a routing algorithm for Spherical Mesh, which guarantees both the balance of workload and a shorter network diameter.

- Finally, we evaluate Spherical Mesh using NS-3. Our simulation results show that Spherical Mesh can decrease maximal routing path by $20 \%$ at most.

The rest of the paper is organized as follows. We briefly review the related work in Section II and present technical preliminaries in Section III. Construction of Spherical Mesh and Routing in this network topology are depicted in Section IV and Section V. In Section VI, we show the performance of Spherical Mesh by simulations. Finally, we conclude this paper in Section VII.

\section{RELATED WORK}

Our work in wireless data center network topology necessarily builds upon work in a number of related areas. Traditional data centers use commodity-class computers and switches which are connected by wires. There are many representative topologies, such as Fattrees [7], DCell [3], BCube [2] and so on.

Meanwhile, with the development of $60 \mathrm{GHz}$ wireless technology, several work begins to use this technology to improve the performance of data center networks. $60 \mathrm{Ghz}$ wireless technology was first proposed to data center networking by Ranachandran et al. [9], as a solution to reduce the cabling complexity. Inspired by this work, many researchers have begun to use wireless links to argument [4], [5] or replace [11], [13] wired links. The most representative work is Flyways [5], which is proposed by Kandula et al. . This work puts antennas on the top of rack and builds a square mesh that is connected by wireless links. Although a number of links are obstructed by antennas, a few additional flyways substantially improve the performance in data center networks.

Recently, an innovative work, 3D beamforming [16], [17], was proposed to solve the problem of link blockage by bouncing off data center ceilings.

\section{PRELIMINARIES}

In this section, we firstly introduce the background of millimeter wavelength wireless technology, and then describe our system model for wireless data center networks.

\section{A. Millimeter Wavelength Wireless Technology}

Spectrum between $57-64 \mathrm{GHz}$, colloquially known as the $60 \mathrm{GHz}$ band, has various advantages as follows:

- $60 \mathrm{GHz}$ technology has huge unlicensed bandwidth. Most countries worldwide have at least $5 \mathrm{GHz}$ of continuous bandwidth available [15]. We can apply this technology for many gigabit wireless applications.

- $60 \mathrm{GHz}$ beamforming radios are becoming more available and affordable. Horn antennas [4] or antenna arrays [12] can use both mechanical and electronic mechanisms to achieve fine-grain directional control [4].

However, the free-space loss is higher due to the small $5 \mathrm{~mm}$ wavelength for $60 \mathrm{GHz}$ wireless signal (Friis law [10]). Channels for $60 \mathrm{GHz}$ are 100 times wider and thus $20 \mathrm{~dB}$ noisier to enable high multi-Gpbs bit-rates for transmissions [1], [14].

To guarantee stable links, indoor $60 \mathrm{GHz}$ wireless technology such as 802.11ad targets a short range of $10 \mathrm{~m}$ [1], which we consider as the effective transmission range of $60 \mathrm{GHz}$ radios.

Moreover, link blockage is a limiting factor for $60 \mathrm{GHz}$ links. Link blockage happens when there is some obstacles in the transmission path. Because of its $5 \mathrm{~mm}$ wavelength, any object larger than $2.5 \mathrm{~mm}$ wavelength can effectively block or reflect wireless signal.

\section{B. System Model}

In wireless data center networks, there are racks equipped with antennas as wireless radios. Suppose the size of network is $m \times n$, every rack in the network is assigned with a tuple value $(i, j)$, which indicates that this rack is in the $i$ th row and $j$ th column. The distance between two adjacent nodes in horizontal (vertical) direction is $l(w)$.

Here we assume that each top-of-rack (ToR) switch can be equipped with a single $60 \mathrm{GHz}$ horn antenna. These antennas are half-duplex and can work on different channels. The number of $60 \mathrm{GHz}$ wireless channels are more than enough to neglect signal interference.

Here we use the network diameter to measure performance in wireless network topology. The metric is affected by the largest distance between nodes in the network first. However, it can be affected by link blockage. On that condition, the actual transmission distance may be less than $r$, which will increase the network diameter. 


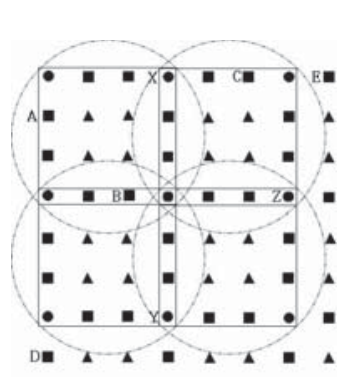

(a) Spherical Mesh

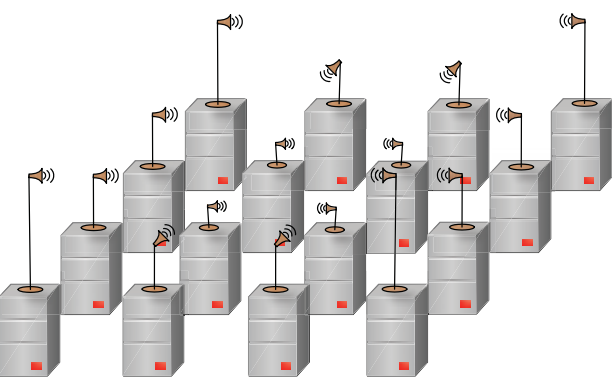

(b) Spherical Mesh Unit

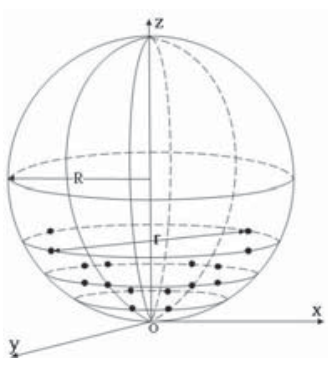

(c) The coordinate system of $S M U$

Figure 1. The structure of Spherical Mesh

We want to achieve two goals in this system. One is to mitigate link blockage without making constraints on the ceilings. The other is to decrease the network diameter. If the network diameter is relatively small, it will decrease the number of hops in routing path.

\section{Spherical Mesh Topology}

In this section, we propose a new topology for wireless data centers, called Spherical Mesh, which leverages the geometric properties of the sphere to mitigate link blockage. We construct a basic component first, and then extend it to the whole network.

\section{A. Spherical Mesh Unit Structure}

Spherical Mesh Unit $(S M U)$ is a basic component in the whole Spherical Mesh. It consists of an array of racks, in which every two nodes can communicate with each other directly. To construct a $S M U$, we put all antennas in the unit on a sphere. Since any three nodes on the sphere are not in the same line, link blockage will not happen. A simple example of $4 \times 4 S M U$ is shown in Figure 1(b).

We want all the nodes in the unit are located on the sphere with a radius of $R$. So we set the center of $S M U$ with a height of top-of-rack as coordinate origin and build a 3-dimensional Rectangular Coordinate System in Figure 1(c), where each black solid point represents an antenna one by one corresponding to Figure 1(b). The coordination of each node in $S M U$ satisfies the following spherical equation.

$$
x^{2}+y^{2}+(z-R)^{2}=R^{2} .
$$

If we use solid points with different shapes represent antennas on the different layers, the whole Spherical Mesh is as what Figure 1(a) depicts.

\section{B. Spherical Mesh Construction}

The whole Spherical Mesh consists of several $S M U$ s. Suppose each $S M U$ consists of $m_{0} \times n_{0}$ racks within the range of $r \times r m^{2}$, we can compute $m_{0}$ and $n_{0}$ by the following equation.

$$
m_{0}=\left\lfloor\frac{\sqrt{2} r}{2 l}\right\rfloor+1, n_{0}=\left\lfloor\frac{\sqrt{2} r}{2 w}\right\rfloor+1
$$

Since we have the size of $S M U$, we can get the relative coordination $\left(x_{i j}, y_{i j}\right)$ for node $(i, j)$ according to Figure 1(c).

$$
\left\{\begin{array}{l}
x_{i j}=\left((i-1) \%\left(m_{0}-1\right)-\frac{m_{0}-1}{2}\right) l \\
y_{i j}=\left((j-1) \%\left(n_{0}-1\right)-\frac{n_{0}-1}{2}\right) w
\end{array}\right.
$$

After we plug $x_{i j}$ and $y_{i j}$ in Equation 1, we have the relative height $h_{i j}$ of the node to the top-of-rack.

$$
h_{i j}=R+\sqrt{R^{2}-x_{i j}^{2}-y_{i j}^{2}}
$$

Finally, we could construct the whole Spherical Mesh by simply placing the antenna in the $i$ th row $j$ th column at the height of $h_{i j}$.

\section{Routing in Spherical Mesh}

In this section, we calculate the network diameter of Spherical Mesh by analyzing its properties. We also propose a routing algorithm in Spherical Mesh which decreases the length of routing path and ensures the balance of workload.

\section{A. Properties of Spherical Mesh}

We classify all the nodes into three categories according to their relative positions for better explanation.

$$
(i-1) \%\left\lfloor\frac{\sqrt{2} r}{2 l}\right\rfloor=0,(j-1) \%\left\lfloor\frac{\sqrt{2} r}{2 w}\right\rfloor=0
$$

- Sphere Nodes. All nodes in the Spherical Mesh can be defined as Sphere nodes. For any two sphere 
nodes in the same $S M U$, they can communicate with each other directly.

- Chord Nodes. Every two adjacent $S M U$ s have a common line of nodes in Figure 1(a). We define these nodes as Chord nodes, which can communicate directly with any nodes in the two units. Chord nodes are special Sphere Nodes, whose ordination satisfy one or both expressions in Equation 5.

- Mesh Nodes. Every four adjacent $S M U$ s have a common node which is in the highest layer in the whole mesh. We define it as Mesh nodes, which can forward signal to any node in the four units. Mesh nodes are special Chord nodes, whose ordinations satisfy both expressions in Equation 5 .

The routing path between two nodes is mainly dependent on their mutual distances. Here we assume the area is $L \times L m^{2}$. Then we can get the following theorem.

Theorem 1 The maximal path length in Spherical Mesh routing is at most $\left\lfloor\frac{\sqrt{2} L}{2 r}\right\rfloor+1$.

Proof: The diagonal of the square area of data center is $\sqrt{2} L$. In each $S M U$, the diameter is equivalent to the transmission range $r$, such that there will be at least $\left\lfloor\frac{\sqrt{2} L}{2 r}\right\rfloor$ Mesh nodes on the transmission path. Consider the most worse condition shown in Figure 1(a). Node $D$ wants to communicate with node $E$. The signal goes through Mesh nodes $Y, Z$ to reach the boundary. Then there should be one more hop to reach node $E$. So the maximal path length in Spherical Mesh routing is $\left\lfloor\frac{\sqrt{2} L}{2 r}\right\rfloor+1$.

Inferred from Theory 1, we have the following corollary.

Corollary 1 The diameter of a Spherical Mesh network is at most $\left\lfloor\frac{\sqrt{2} L}{2 r}\right\rfloor+1$.

Corollary 1 shows that, the network diameter of Spherical Mesh is linear to the length of the data center.

\section{B. Spherical Mesh Routing Algorithm}

Derived by Theorem 1 and Corollary 1, we propose a simple and efficient routing algorithm. It consists of Mesh routing algorithm, Sphere routing algorithm and Chord routing algorithm.

Sphere routing algorithm works on the source node and destination node. If both nodes are on the same $S M U$, the algorithm can detect this condition and set up a direct link between them. If they are not on the same sphere, it can find the Mesh node nearest to the destination.

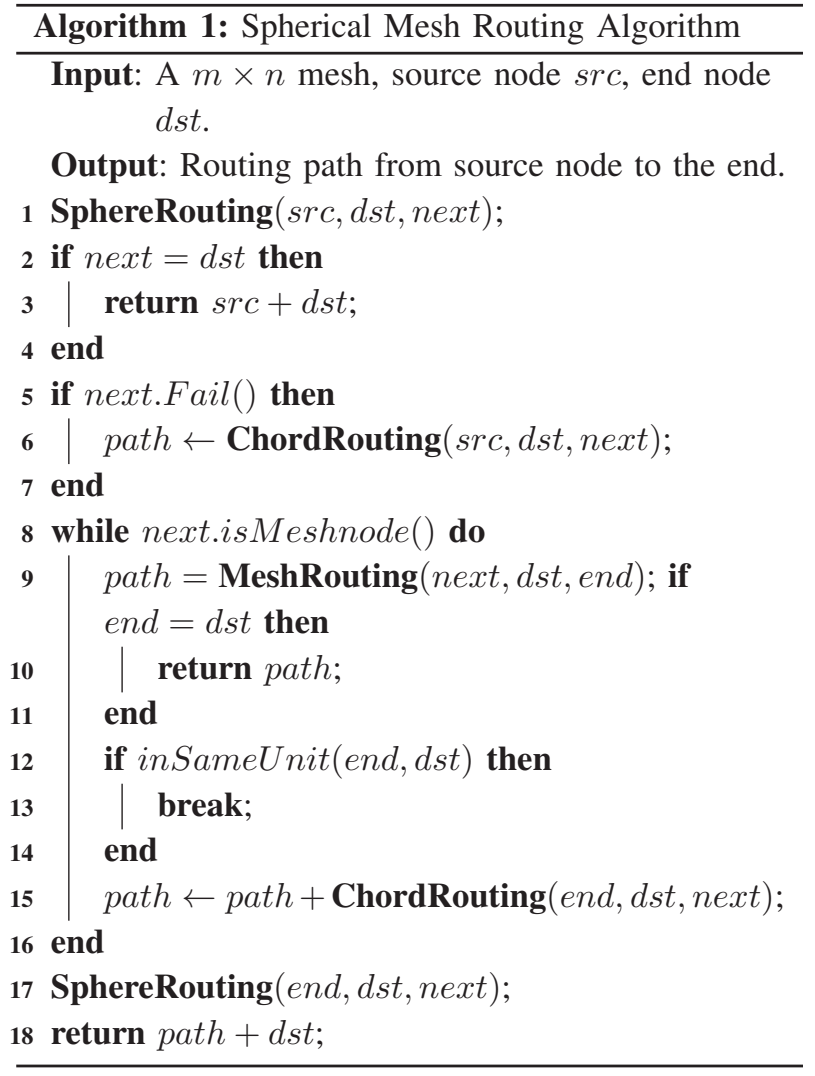

Mesh routing algorithm works to find a shortest path from one Mesh node to the other Mesh node which is nearest to the destination. This is similar to routing in mesh-connected arrays [6]. But our routing algorithm allows one node to transmit the signal to its diagonal node by utilizing wireless communication technology.

However, Mesh nodes are easy to become hotspots. To implement the balance of workload, we use Chord routing algorithm to share the excess network traffic. The Chord routing algorithm works when Sphere routing and Mesh routing fail to find a Mesh node nearest to the destination. In that case, it continues to find a proper Chord node and forward signal to it until an appropriated Mesh node is available. It also stops when the Chord node is in the same unit with the destination.

The whole algorithm works as what Algorithm 1 shows. First, by Sphere routing, the start node can send the signal to the destination or forward it to the nearest Mesh node. If the mesh node is busy or failed, Chord routing will help find a path towards a Mesh node or the Chord node nearest to the destination. When the signal is forwarded to a Mesh node, Mesh routing will find the Mesh node nearest to the destination if available. Finally, the signal is transmitted to the destination from 


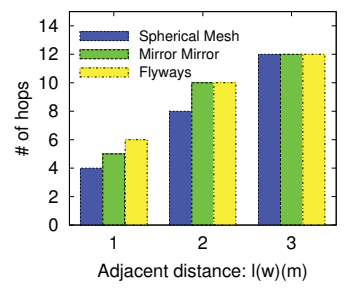

(a) Same Scale

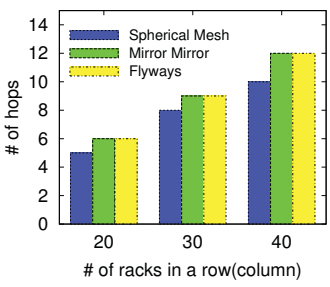

(b) Different Scales
Figure 2. Network performance of Spherical Mesh

a Mesh or Chord node by Sphere routing.

\section{Simulation}

In this section, we exhibit the performance of Spherical Mesh. The simulation is implemented in NS-3 [8].

We implement Spherical Mesh based on square mesh topology. Racks are put in a grid with equivalent adjacent distance $l$ horizontally and $w$ vertically. The network performance of Spherical Mesh is measured by the decrease of the network diameter. Here, we choose the number of hops of routing in the diameter as our metric, since it is proportional to the length of diameter. We compare this metric with two representative topologies, Flyways [5] and 3D beamforming [17]. According to most situations in data centers, we assume the ceiling height $h$ of data center is $3 \mathrm{~m}$. Our simulations consist of the following two groups.

The first group is that the size of data center is set to be $25 \times 25 . l$ and $w$ are equivalent and range from $1.0 \mathrm{~m}$ to $3.0 \mathrm{~m}$. The result is shown in Figure 2(a).

The other is that the size of data center ranges from $20 \times 20$ to $40 \times 40$ evenly. Both $l$ and $w$ are set to be $1.5 \mathrm{~m}$. The result is shown in Figure 2(b).

We find that Spherical Mesh have shorter routing path than Flyways and 3D beamforming in both Figure 2(a) and Figure 2(b). This is because Flyways cannot always forward signal to the next node which is nearest to the destination. In order to avoid link blockage, the procedure of routing has more jumps and often performs not well. 3D beamforming mitigates link blockage, but bouncing off high ceilings will increase the routing path somehow.

Spherical Mesh can minimize the routing path and guarantee balance of workload. When the density of racks or the size of data center is larger, it will perform better and have a decrease about $20 \%$ in routing path. But we also notice that, when the arrangement of racks is sparse, its performance is also no worse than the other two topologies in Figure 2(a). So Spherical Mesh achieves our goals.

\section{CONCLUSION}

In this paper, we have presented the design, analysis and implementation of Spherical Mesh. Spherical Mesh is a flexible network topology to interconnect servers with wireless links. Our insight is to put $60 \mathrm{GHz}$ antennas on identical spheres. We can mitigate link blockage and decrease the network diameter. Our routing algorithm on Spherical Mesh achieves minimal routing distance and guarantees the balance of workload.

Although wired networks will exist in a fairly long time, we believe that our Spherical Mesh can improve the performance of a broad range of data center deployments. A straightforward extension is to use more intelligent antennas to save the rotation time of horns. We leave such extensions as the future work.

\section{REFERENCES}

[1] IEEE P802.11ad/D9.0: Draft Standard for Information Technology -Enhancements for Very High Throughput in the $60 \mathrm{GHz}$ Band.

[2] C. Guo, G. Lu, D. Li, H. Wu, X. Zhang, Y. Shi, C. Tian, Y. Zhang, and S. Lu. BCube: a high performance, server-centric network architecture for modular data centers. In SIGCOMM, 2009.

[3] C. Guo, H. Wu, K. Tan, L. Shi, Y. Zhang, and S. Lu. DCell: a scalable and fault-tolerant network structure for data centers. In SIGCOMM, 2008.

[4] D. Halperin, S. Kandula, J. Padhye, P. Bahl, and D. Wetherall. Augmenting data center networks with multi-gigabit wireless links. In SIGCOMM, 2011.

[5] S. Kandula, J. Padhye, and P. Bahl. Flyways to de-congest data center networks. In HotNets, 2009.

[6] M. Kunde. Routing and sorting on mesh-connected arrays. In VLSI Algorithms and Architectures, pages 423-433. Springer, 1988.

[7] C. E. Leiserson. Fat-trees: universal networks for hardwareefficient supercomputing. IEEE Transactions on Computers, 100(10):892-901, 1985.

[8] NS-3. http://www.nsnam.org/.

[9] K. Ramachandran, R. Kokku, R. Mahindra, and S. Rangarajan. $60 \mathrm{GHz}$ data-center networking: wireless $\rightarrow$ worryless? NEC Laboratories America, Inc., Tech. Rep., July, 2008.

[10] T. S. Rappaport. Wireless communications: principles and practice. Publishing House of Electronics Industry, 2004.

[11] J.-Y. Shin, E. G. Sirer, H. Weatherspoon, and D. Kirovski. On the feasibility of completely wireless datacenters. In ANCS, 2012.

[12] SiBeam. http://sibeam.com/whitepapers/.

[13] H. Vardhan, N. Thomas, S.-R. Ryu, B. Banerjee, and R. Prakash. Wireless data center with millimeter wave network. In GLOBECOM, 2010.

[14] WirelessHD. http://wirelesshd.org/.

[15] S.-K. Yong, P. Xia, and A. Valdes-Garcia. 60GHz Technology for Gbps WLAN and WPAN: From Theory to Practice. Wiley. com, 2011.

[16] W. Zhang, X. Zhou, L. Yang, Z. Zhang, B. Y. Zhao, and H. Zheng. 3D beamforming for wireless data centers. In HotNets, 2011.

[17] X. Zhou, Z. Zhang, Y. Zhu, Y. Li, S. Kumar, A. Vahdat, B. Y. Zhao, and H. Zheng. Mirror mirror on the ceiling: flexible wireless links for data centers. In SIGCOMM, 2012. 